CASO CLÍNICO

\title{
Cierre de fístula gastrocutánea persistente posterior a retiro de PEG: un as debajo de la manga
}

\author{
José Vargas-Jiménez ${ }^{1 *}$ y Ana Lorena Madrigal-Méndez ${ }^{2}$ \\ ${ }^{1}$ Gastroenterología y Endoscopia Digestiva; ${ }^{2}$ Gastroenteróloga, Servicio de Gastroenterología y Endoscopia Digestiva, Hospital San Juan de \\ Dios, San José, Costa Rica
}

\section{Resumen}

La colocación de sondas de gastrostomía percutánea endoscópica (PEG) es un procedimiento común. De manera infrecuente, a la retirada del PEG se da la persistencia del trayecto fistuloso con el desarrollo de fístula gastrocutánea. Reportamos el caso de cierre endoscópico de una fístula gastrocutánea mediante el cierre en tulipán en una mujer de 69 años.

Palabras clave: Fístula gastrocutánea. Cierre en tulipán. Endoloop ${ }^{\oplus}$ Gastrostomía percutánea endoscópica. PEG.

\section{Closing of persistent gastrocutaneous fistula after PEG removal: an ace under the sleeve}

\section{Abstract}

Percutaneous endoscopic gastrostomy (PEG) tube is a commonly performed procedure. Upon removal of PEG tube, persistent fistulous tract ensues with the subsequent development of gastrocutaneous fistula. We report the case of an endoscopic closure of a gastrocutaneous fistula using tulip bundle technique on a 69 year-old-woman.

Key words: Gastrocutaneous fistula. Tulip bundle closure. Endoloop ${ }^{\circ}$. Percutaneous endoscopic gastrostomy. PEG.

\section{Correspondencia:}

*José Vargas-Jiménez

Servicio de Gastroenterología y Endoscopia

Digestiva

Hospital San Juan de Dios

Paseo Colón

Fecha de recepción: 09-01-2019

C.P. 10105, San José, Costa Rica

E-mail: jovargasjimenez @ gmail.com
Fecha de aceptación: 28-02-2019

DOI: 10.24875/END.M19000006
Disponible en internet: 06-05-2019 Endoscopia. 2019;31:90-92 www.endoscopia-ameg.com 0188-9893/C 2019. Asociación Mexicana de Endoscopia Gastrointestinal, publicado por Permanyer México SA de CV, todos los derechos reservados. 


\section{Introducción}

Las sondas de gastrostomía percutánea endoscópica (PEG) fueron introducidas por Gauderer en el año $1980^{1}$ y desde su introducción se han convertido en un procedimiento frecuente, realizado en aquellos que ameritan nutrición enteral prolongada.

Se han descrito múltiples complicaciones, tanto mayores como menores ${ }^{2,3}$; entre ellas se incluyen el síndrome del botón enterrado, infección, fuga periostomal y perforación colónica, entre otras.

La falla al cierre espontáneo del trayecto fistuloso, posterior a la retirada de la sonda de PEG, tiene como consecuencia el desarrollo de una complicación poco usual: la fístula gastrocutánea.

Se han descrito múltiples terapias para el tratamiento de esta complicación, entre ellas: el uso de coagulación con argón, uso de clips endoscópicos, técnicas de sutura endoscópica y cirugía, entre otras. Sin embargo, el éxito de estas terapias está lejos de ser el óptimo.

\section{Resumen de caso}

Una paciente de 69 años, conocida diabética e hipertensa y con secuelas de un evento cerebrovascular total de la circulación anterior, desarrolla una fístula gastrocutánea posterior a la retirada (1 mes) de una sonda de gastrostomía percutánea.

Dados los antecedentes de la paciente se decide realizar cierra del trayecto fistuloso por medio de vía endoscópica.

Mediante endoscopia se identificó el oricio interno de la fístula; inicialmente se intentó realizar el cierre utilizando un clip sobre el endoscopio (OTSC ${ }^{\circledR}$, over the scope clip; Ovesco Endoscopy, Tübingen, Alemania). Sin embargo la liberación de este fue defectuosa, por lo que se decidió utilizar la técnica de cierre en tulipán ${ }^{4}$ para el manejo de esta complicación.

Se utilizó un endoscopio de doble canal (GIF-2T160; Olympus, Tokyo, Japón) en conjunto con un Endoloop ${ }^{\circledR}$ de 20 mm (MAJ-340; Olympus). Inicialmente fijamos el Endoloop ${ }^{\circledR}$ en la mucosa normal que rodea el defecto, de distal a proximal, a una distancia aproximada de $5 \mathrm{~mm}$ de los bordes del defecto (Fig. 1). En total se colocaron seis clips (M00522610; Resolution Clip; Boston Scientific) para la fijación adecuada del Endoloop ${ }^{\circledR}$, y posteriormente se procedió a realizar el cierre de este, lo cual generó un cierre completo del defecto (Fig. 2). Finalmente se corroboró el cierre del trayecto fistuloso, sin observar fuga de aire o líquido. A las

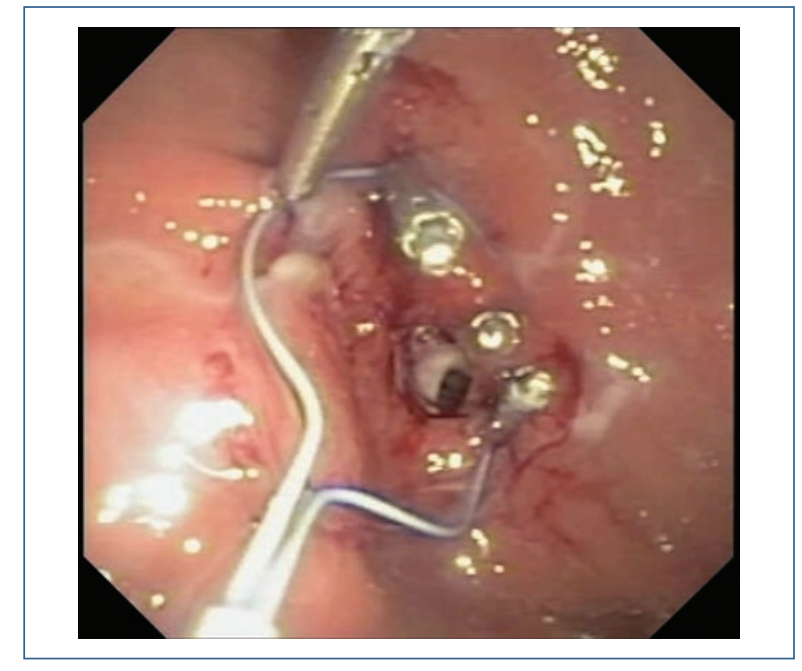

Figura 1. Orificio gástrico de la fístula, con Endoloop ${ }^{\circledR}$ parcialmente fijado con endoclips alrededor de los bordes de la fístula.

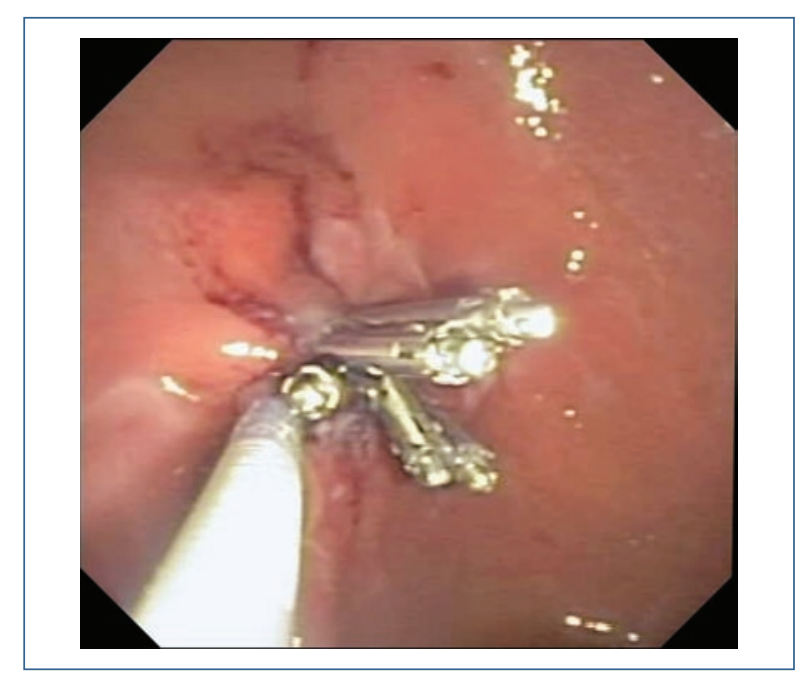

Figura 2. Apariencia al completar el cierre del Endoloop ${ }^{\circledR}$, obteniendo un cierre completo de la fístula.

cuatro semanas de seguimiento se continúa corroborando el cierre completo de la fístula gastrocutánea.

\section{Discusión}

Las fístulas gastrocutáneas desarrolladas posteriormente a la retirada de sondas percutáneas de gastrostomía son complicaciones poco frecuentes y con múltiples manejos descritos, muchos de estos con una respuesta subóptima. Es importante recordar que la mayoría de los pacientes que son sometidos a 
colocación de sonda tipo PEG son pacientes adultos mayores con múltiples comorbilidades, por lo que manejos mínimamente invasivos deberán ser siempre la primera línea de tratamiento.

La técnica anteriormente descrita y sus diferentes modificaciones ${ }^{4,5}$ se ha utilizado en perforaciones colónicas agudas y en fístulas persistentes, y ha probado ser una terapia efectiva.

Sin embargo, a la hora de determinar cuál de todas las terapias endoscópicas es la más adecuada, es importante tomar en cuenta la temporalidad de la fístula, ya que a mayor tiempo de evolución la fibrosis será más importante, lo que aumentará la probabilidad de fallo al tratamiento. En estos casos la desvitalización del tejido del trayecto fistuloso y circundante al orificio interno es de vital importancia; esta se puede realizar con curetaje, pinzas de biopsia o más comúnmente con el uso de plasma argón. En el caso presentado, se tomó la decisión de no realizar desvitalización del tejido, dado el poco tiempo de evolución de la fístula.

Otro punto importante para tomar en cuenta al utilizar la técnica con Endoloop ${ }^{\circledR}$ es realizar de manera muy lenta el cierre y aproximación del defecto, para evitar así el desprendimiento de los clips utilizados para la fijación del Endoloop ${ }^{\circledR}$.

A manera de conclusión, la técnica utilizada anteriormente ha mostrado ser una opción efectiva, segura y reproducible, por lo que deberá ser parte de nuestro arsenal terapéutico para el manejo de esta inusual complicación.

\section{Declaración de conflictos de interés}

Ninguno de los autores presentan conflictos de intereses ni apoyos financieros relacionados con esta publicación.

\section{Bibliografía}

1. Gauderer MW, Ponsky JL, Izant RJ. Gastrostomy without laparotomy: a percutaneous endoscopic technique. J Pediatr Surg. 1980;15(6):872-5.

2. Sealock RJ, Munot K. Common gastrostomy feeding tube complications and troubleshooting. Clin Gastroenterol Hepatol. 2018;16(12):1864-9.

3. Shellito PC, Malt RA. Tube gastrostomy. Techniques and complications. Ann Surg. 1985;201(2):180-5.

4. Perri F, Gentile M, Scimeca D, Terracciano F, Merla A, Spirito F, et al Closure of a gastrocutaneous fístula by a tulip-bundle technique. Endoscopy. 2011;43(S 02):E419.

5. Mocciaro F, Curcio G, Tarantino I, Barresi L, Spada M, Petri SL, et al. Tulip bundle technique and fibrin glue injection: Unusual treatment of colonic perforation. World J Gastroenterol. 2011;17(8):1088-90. 